\title{
Modeling non-perturbative field-driven vibronic dynamics: Selective state preparation and nonlinear spectroscopy
}

\author{
Supporting Information
}

\section{This PDF includes:}

Justification of RWA in this application

Wigner spectrum of electromagnetic field

PLDM field-driven method vs exact calculations

How to prepare chirped pulses with fixed $|E(\Omega)|^{2}$

Heat maps for ground state vibrational excitation as a function of driving chirped pulse field parameters with different carrier frequencies

Comparison of pump-probe spectra of the simple harmonic model at $\mathrm{T}=77 \mathrm{~K}$ and $\mathrm{T}=300 \mathrm{~K}$

\section{S1 Justification of RWA}

The Rotating Wave Approximation (RWA) is often introduced as an accurate approximation to the field-matter interaction term at frequencies resonant with electronic transitions. ${ }^{1}$ Additionally, invoking the RWA allows a more simple analysis while making connection to experimental spectroscopic signals appearing in phase-matched directions. ${ }^{2,3}$ We start by writing the oscillatory term from the incident field in complex form as $\cos (\omega t)=\frac{1}{2}(\exp (i \omega t)+\exp (-i \omega t))$, where we have assumed for simplicity that $\varphi(t)=0$. From here, a rationale for the RWA is most easily seen in the interaction representation, ${ }^{4}$ where the time-dependent field-matter interaction term in the Hamiltonian takes the form $\hat{H}_{F}^{I}(t)=\exp \left(\frac{i}{\hbar} \hat{H}_{\text {mol }} t\right) \hat{H}_{F}(t) \exp \left(\frac{-i}{\hbar} \hat{H}_{\text {mol }} t\right)$. For a simple two-level system (consisting of a "ground" and "excited" state) where the states are coupled only through the incident radiation field, the interaction representation of this operator takes the form

$$
\begin{aligned}
\hat{H}_{F}^{I}(t) & =-\frac{1}{2} \boldsymbol{\mu}_{g e} \cdot \boldsymbol{\epsilon} \tilde{E}(t)\left(\exp \left(i \omega_{c} t\right)+\exp \left(-i \omega_{c} t\right)\right)\left[|g\rangle\left\langle e\left|e^{-i \omega_{e g} t}+\right| e\right\rangle\langle g| e^{i \omega_{e g} t}\right] \\
& =-\frac{1}{2} \boldsymbol{\mu}_{g e} \cdot \boldsymbol{\epsilon} \tilde{E}(t)|g\rangle\langle e|\left[e^{-i\left(\omega_{e g}+\omega_{c}\right) t}+e^{-i\left(\omega_{e g}-\omega_{c}\right) t}\right]+\text { h.c. }
\end{aligned}
$$

where $\omega_{e g}=\left(E_{e}-E_{g}\right) / \hbar, \omega_{c}$ is the carrier frequency of the incident radiation, and $\tilde{E}(t)$ is the real valued temporal envelope of the field.

When we are considering high frequency incident optical radiation fields that have carrier frequencies near resonant with the molecular transition frequency (i.e. $\omega_{c} \approx \omega_{e g}$ ), the operator can be approximated as 


$$
\hat{H}_{F}^{I}(t) \approx-\frac{1}{2} \boldsymbol{\mu}_{g e} \cdot \boldsymbol{\epsilon} \tilde{E}(t)|g\rangle\langle e|\left[e^{-2 i \omega_{e g} t}+1\right]+\text { h.c. }
$$

Where h.c. stands for the Hermitian conjugate of the terms appearing to the left. The RWA corresponds to ignoring the rapidly oscillating term $\left(\propto e^{-2 i \omega_{e g} t}\right)$ that contributes little to the comparatively slow dynamics of the near resonant term. Rotating back into the Schrödinger representation, the RWA approximation to the field-matter interaction term of the Hamiltonian is defined as

$$
\hat{H}_{F}^{\mathrm{RWA}}(t)=-\frac{1}{2} \boldsymbol{\mu}_{g e} \cdot \boldsymbol{\epsilon} \tilde{E}(t)\left[e^{i \omega_{c} t}|g\rangle\left\langle e\left|+e^{-i \omega_{c} t}\right| e\right\rangle\langle g|\right]
$$

As seen in Figure S1, the RWA is a good approximation in the case of electronic excitations of chromophores that occur with excitation frequencies generally on the order of $10^{4} \mathrm{~cm}^{-1}$ when the incident field has a carrier frequency, $\omega_{c}$, near resonant with the electronic transition.

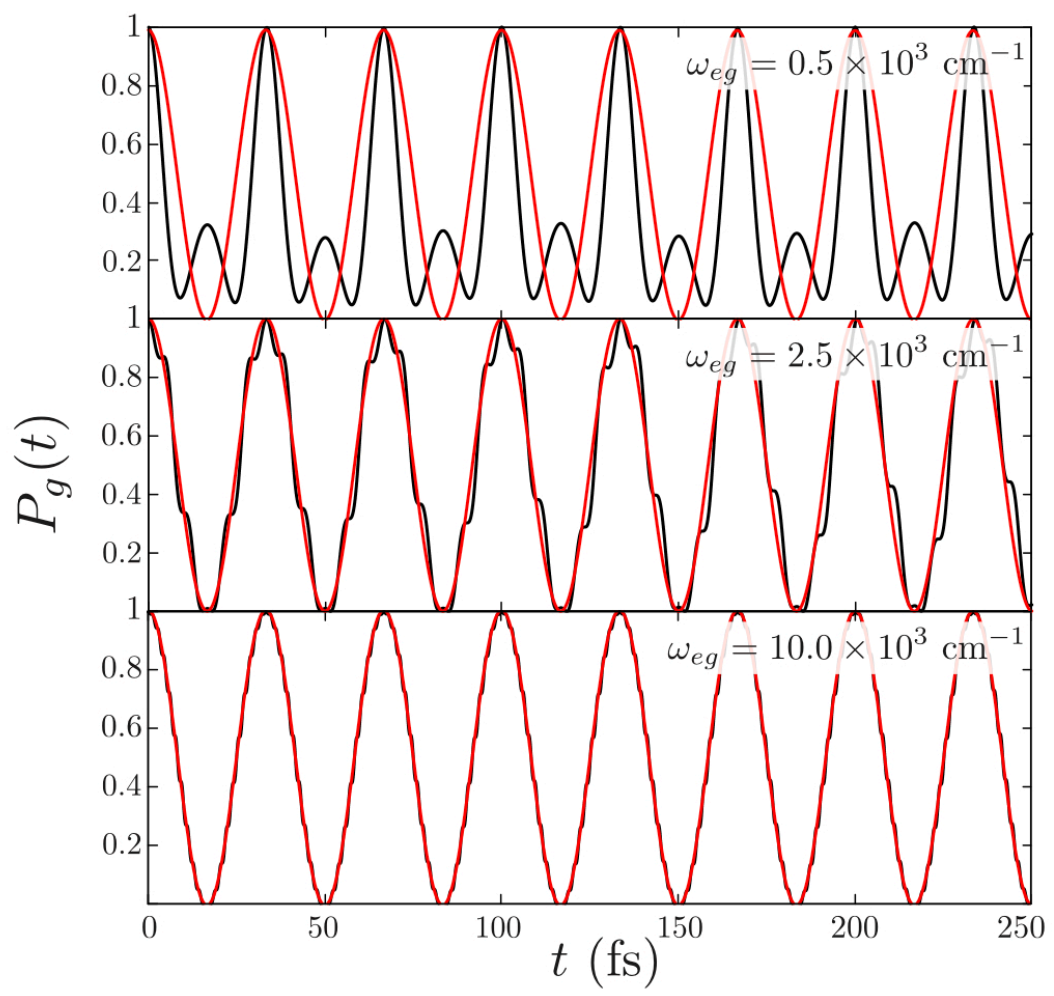

Figure S1: Ground state population dynamics of a two level system coupled through an incident radiation field. Here, the excitation frequency is chosen to be resonant with the incident radiation such that there is a complete population inversion. The electric field is chosen to have a constant temporal envelope such that the field-matter coupling term in the Hamiltonian takes the form $\hat{H}_{F}(t)=-\hat{\boldsymbol{\mu}} \cdot \boldsymbol{E}(t)=$ $1000 \mathrm{~cm}^{-1} \times \cos \left(\omega_{e g} t\right)$, while the molecular Hamiltonian is defined by $E_{e}-E_{g}=\hbar \omega_{e g}$. In the above plots, the red line represents the RWA solution, while the black line represents the full solution. The approximate RWA solution is shown to converge to the full solution as the frequency is increased.

Since the RWA approximation for field-matter interactions that induce electronic excitations provides an accurate representation of the photo-induced excitation, as shown 
in Figure S1, we are in position to implement this approximation within the PLDM approximation to the time-evolved density matrix. Since the PLDM algorithm relies on numerically integrating Hamilton's equations for all DOFs, the time-dependent fieldmatter interaction term must be updated at each time point along the trajectory. Luckily, because we are interested in electronic excitations that occur with frequencies that align well with the RWA, we can arbitrarily shift the excitation frequency (and field carrier frequency) down while implementing the RWA to a value that allows us to minimize the number of time integration steps (effectively artificially increasing our required minimal time step for accurate numerical integration of Hamilton's equations) to reduce the computational effort required to obtain a trajectory without sacrificing any of the dynamics that we would observe without this arbitrary shift had we not made the RWA. This can be seen by noticing that the RWA results presented in Figure S1 provide the same result regardless of the value of $\omega_{e g}$, while the full result only converges to the RWA result as the value of $\omega_{e g}$ approaches a value that is representative of a typical electronic excitation in a chromophore. The ability to perform this arbitrary shift, of course, assumes that the electric field is the exclusive source of coupling between the ground and excited state manifolds.

\section{S2 Wigner Spectrum}

The Wigner spectrum of the electric field maintains the properties that

$$
\frac{1}{2 \pi} \int_{-\infty}^{\infty} d \Omega W(t, \Omega)=|E(t)|^{2}
$$

and

$$
\int_{-\infty}^{\infty} d t W(t, \Omega)=|\tilde{E}(\Omega)|^{2}
$$

Moreover, the time-dependent expectation value of the frequency can be extracted from the Wigner spectrum, resulting in

$$
\omega(t)=\frac{\int_{-\infty}^{\infty} d \Omega \Omega W(t, \Omega)}{\int_{-\infty}^{\infty} d \Omega W(t, \Omega)} \approx \omega_{c}+\dot{\varphi}(t)
$$

which follows from substituting the definition of $E_{+}(t)$ (Equation 8 of the main text) into the expression above. This approximate equality becomes rigorous in the case that $\varphi(t)$ is a quadratic function of function of $t$, which is representative of a linearly chirped pulse. In pulses with ultrashort temporal envelopes this definition of the time-dependent frequency can also be seen by a temporal variation of the phase of the complex field. ${ }^{5}$ 


\section{S3 PLDM field-driven method vs exact calculations}
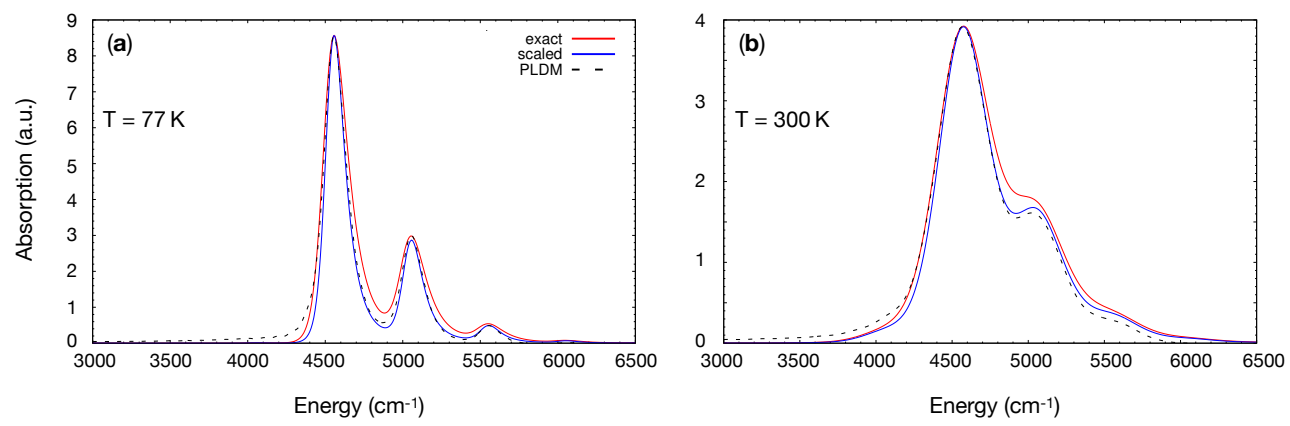

Figure S2: Comparison of linear absorption spectra at (a) $77 \mathrm{~K}$ and (b) $300 \mathrm{~K}$ for the method developed in this paper (PLDM, black dashed curve) and for exact results (red and blue curves). The blue curve is also the result of an exact calculation in which the bath reorganization energy has been scaled to match the PLDM result (by 0.7 for the $77 \mathrm{~K}$ and by 0.85 for the $300 \mathrm{~K}$ spectra).

In order to access the accuracy of the developed method, we compared linear and pump-probe spectra produced with it against exact results. These last were obtained for the studied spin-boson model coupled to a spectral density via the Spectron code. ${ }^{2}$ The results are reported in Figures S2 and S3.

The system is again a simple vibronic model with two electronic wells, a vibrational frequency of $500 \mathrm{~cm}^{-1}$, a Huang-Rhys factor $S=0.35$ and temperature either set to 77 or to $300 \mathrm{~K}$. The system is coupled to a Drude-Lorentz bath with reorganization energy $\lambda=100 \mathrm{~cm}^{-1}$ and $\gamma=100 \mathrm{~cm}^{-1}$. The explicit field employed in the PLDM dynamics was chosen as a very short unchirped gaussian pulse $\left(\sigma_{t}=0.5 \mathrm{fs}\right.$, delta-like in time) whose intensity was kept low enough to allow direct comparison with Spectron response function based results (weak field regime).

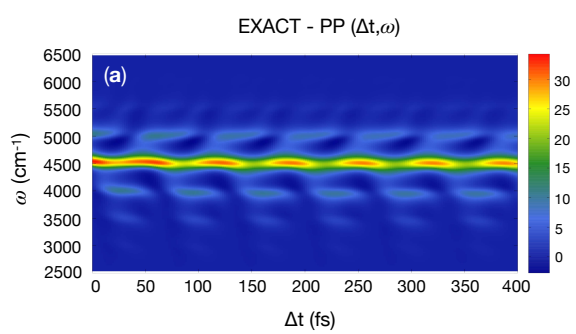

EXACT - FTPP $\left(\omega_{2}, \omega\right)$

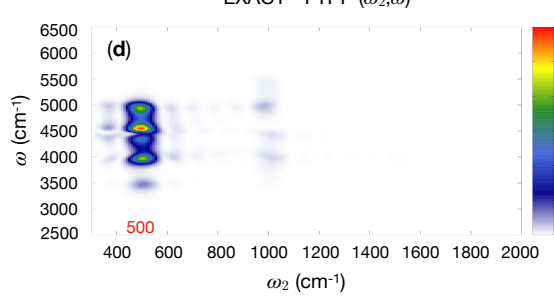

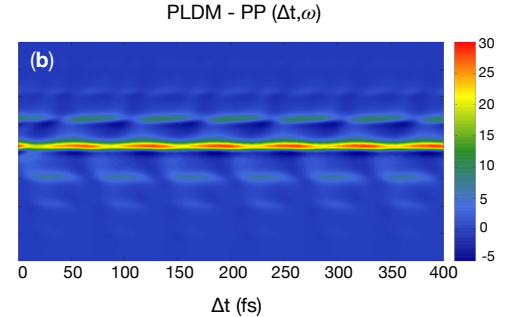

$\operatorname{PLDM}-\operatorname{FTPP}\left(\omega_{2}, \omega\right)$

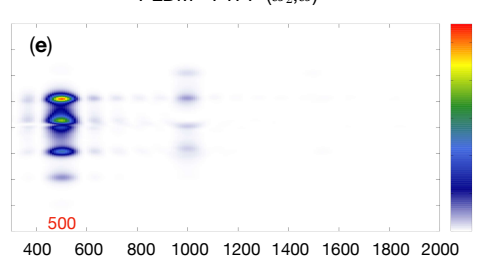

$\omega_{2}\left(\mathrm{~cm}^{-1}\right)$

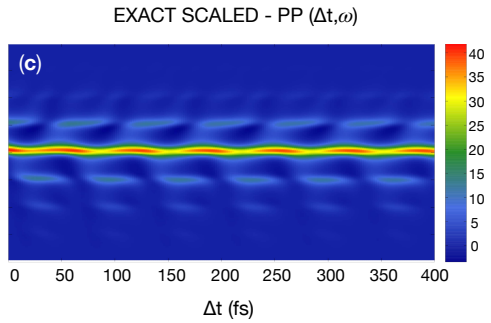

EXACT SCALED - FTPP $\left(\omega_{2}, \omega\right)$

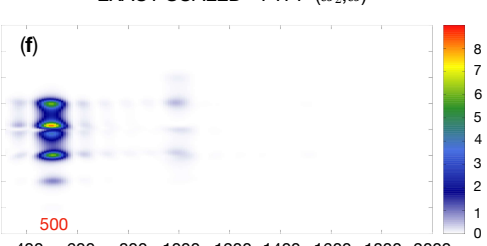

$400 \quad 600 \quad 8001000 \quad 12001400160018002000$ $\omega_{2}\left(\mathrm{~cm}^{-1}\right)$

Figure S3: Comparison of pump-probe maps (a-c) and their power spectra (d-f) for exact and PLDM calculations. All simulations were performed at 77K. The "scaled" calculation was performed by scaling the bath reorganization energy by 0.7 (as for the linear absorption). 
Both linear and non-linear spectra computed with PLDM+explicit field compare well with the exact counterparts, the main difference being a reduced broadening of the former. This reduced broadening comes from the use of focusing in the PLDM algorithm, which has been shown to underestimate decoherence in spin-boson models. ${ }^{6}$ For this reason we have also add the comparison with exact computations in which the bath reorganization energy was scaled by a small amount until matching with the PLDM approach was reached.

In non-linear spectra both the pump-probe and the power spectra maps show a remarkable agreement between the two methods. Again, the PLDM map is less broad than the exact map when both are coupled to the same spectral density. The power spectra shows the same peak structure and shape, both at 500 and $1000 \mathrm{~cm}^{-1}$ (overtone), with the PLDM spectra having a slight stronger intensity of the bluest peak.

\section{S4 How to prepare chirped pulses with fixed $|E(\Omega)|^{2}$}

We start from the notion that in the experiments, the chirp is realized by delaying certain frequency components of a transform limited pulse with respect to others. Therefore, one has to impose that the number of photons of each color is the same as that of the transform limited pulse, which means:

$$
\left|E_{T L}(\Omega)\right|^{2}=\left|E_{\text {chirped }}(\Omega)\right|^{2}
$$

Let us consider a chirped Gaussian pulse written as

$$
E(t)=\lambda e^{-\frac{t^{2}}{2 \sigma^{2}}} e^{i \omega_{c} t} e^{i a t^{2}}
$$

where $a$ is the chirp parameter. The Fourier transform of $E(t)$ is given by

$$
E(\Omega)=\int_{-\infty}^{\infty} E(t) e^{-i \Omega t} d t=\frac{\lambda}{\sqrt{2 A}} e^{-\frac{\left(\Omega-\omega_{c}\right)^{2}}{4 A}}
$$

where $A$ is the complex number given by $A=\frac{1}{2 \sigma^{2}}-i a$.

$|E(\Omega)|^{2}$ is then given by

$$
|E(\Omega)|^{2}=\lambda^{2} \frac{\sigma^{2}}{\sqrt{1+4 \sigma^{4} a^{2}}} e^{-\frac{\left(\Omega-\omega_{c}\right)^{2} \sigma^{2}}{1+4 \sigma^{4} a^{2}}}
$$

(note that since $\sigma$ is in fs and $a$ is in $\mathrm{fs}^{-2}$, the product $\sigma^{4} a^{2}$ is unitless). We now want to make use of Eq. 7, considering that $\left|E_{T L}(\Omega)\right|^{2}=\lambda_{T L}^{2} \sigma_{T L}^{2} e^{-\left(\Omega-\omega_{c}^{T L}\right)^{2} \sigma_{T L}^{2}}$, so that

$$
\lambda_{T L}^{2} \sigma_{T L}^{2} e^{-\left(\Omega-\omega_{c}^{T L}\right)^{2} \sigma_{T L}^{2}}=\lambda^{\prime 2} \frac{\sigma^{\prime 2}}{\sqrt{1+4 \sigma^{\prime 4} a^{2}}} e^{-\frac{\left(\Omega-\omega_{c}\right)^{2} \sigma^{\prime 2}}{1+4 \sigma^{\prime 4} a^{2}}}
$$

which we can translate into a set of three equations, one by equating the pre-exponential intensity terms, and two by equating different parts of the exponent (frequency dependence and width), giving:

$$
\begin{aligned}
\lambda_{T L}^{2} \sigma_{T L}^{2} & =\lambda^{\prime 2} \frac{\sigma^{\prime 2}}{\sqrt{1+4 \sigma^{\prime 4} a^{2}}} \\
\sigma_{T L}^{2} & =\frac{\sigma^{\prime 2}}{1+4 \sigma^{\prime 4} a^{2}} \\
\omega_{c}^{T L} & =\omega_{c}
\end{aligned}
$$


The third equation is satisfied by imposing that the chirped field has the same central frequency as the TL field. By solving the second equation for $\sigma^{\prime 2}$ one get

$$
\sigma_{+/-}^{\prime}=\sigma_{T L} \sqrt{\frac{1 \pm \sqrt{1-16 \sigma_{T L}^{4} a^{2}}}{8 \sigma_{T L}^{4} a^{2}}}
$$

Note that (i) one obtains two possible solutions for $\sigma^{\prime}$, both having the same frequency profile as the transform limited pulse, and (ii) the solutions are real when $1 \geq 16 \sigma_{T L}^{4} a^{2}$ (which means that, given the width of the TL pulse $\sigma_{T L}$, we cannot choose arbitrary values for the chirp parameter, $a$, if we want to retrieve the same shape of the electric field in the frequency domain). One also has two solutions for the intensity of the field in the frequency domain $\left(\lambda^{\prime}\right)$, given by:

$$
\lambda_{+/-}^{\prime}=\lambda_{T L} \frac{\sigma_{T L}}{\sigma_{+/-}^{\prime}}\left(1+4 \sigma_{+/-}^{\prime 4} a^{2}\right)^{\frac{1}{4}}
$$

The fields characterized by $\sigma_{+}^{\prime}\left(\sigma_{-}^{\prime}\right)$ and $\lambda_{+}^{\prime}\left(\lambda_{-}^{\prime}\right)$ are labeled with $L(S)$ in the main text, because they correspond to the solution with longer (shorter) chirped pulse time-profiles.

Figure S4 shows the Wigner plots for the pulses reported in Table 1 of the main text.
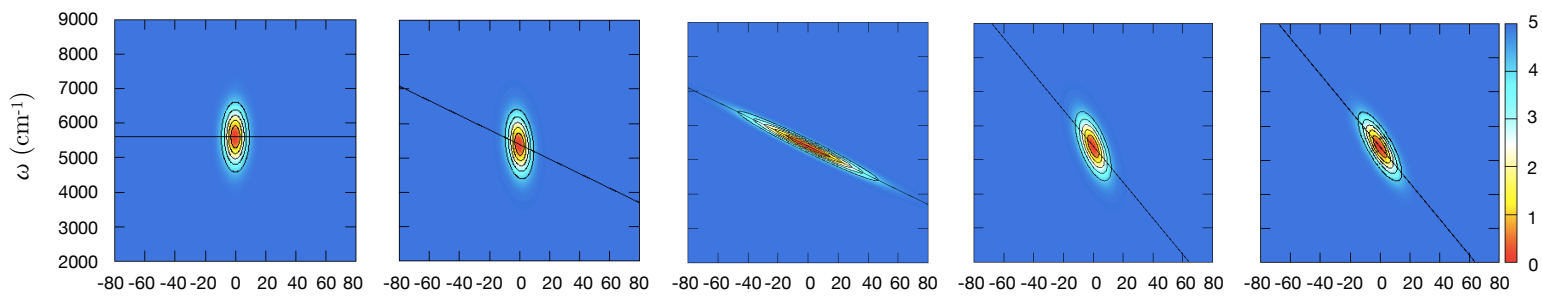

$t(\mathrm{fs})$

Figure S4: Wigner plots for the (a) TL, (b) $\mathrm{C} 1_{S}$, (c) $\mathrm{C} 1_{L}$, (d) $\mathrm{C} 2_{S}$ and (e) $\mathrm{C} 2_{L}$ pulses. The slope of the black line represents the chirp (in $\mathrm{cm}^{-1} / \mathrm{fs}$ ).

\section{S5 Ground state vibrational excitation with different carrier frequencies}

Here we reproduce scans over chirped Gaussian driving field parameters similar to that presented in Figure 2(a) of the main text. Here, however, we show how the heat maps vary for a range of different field carrier frequencies. Note that each heat map has its own color range definition. 

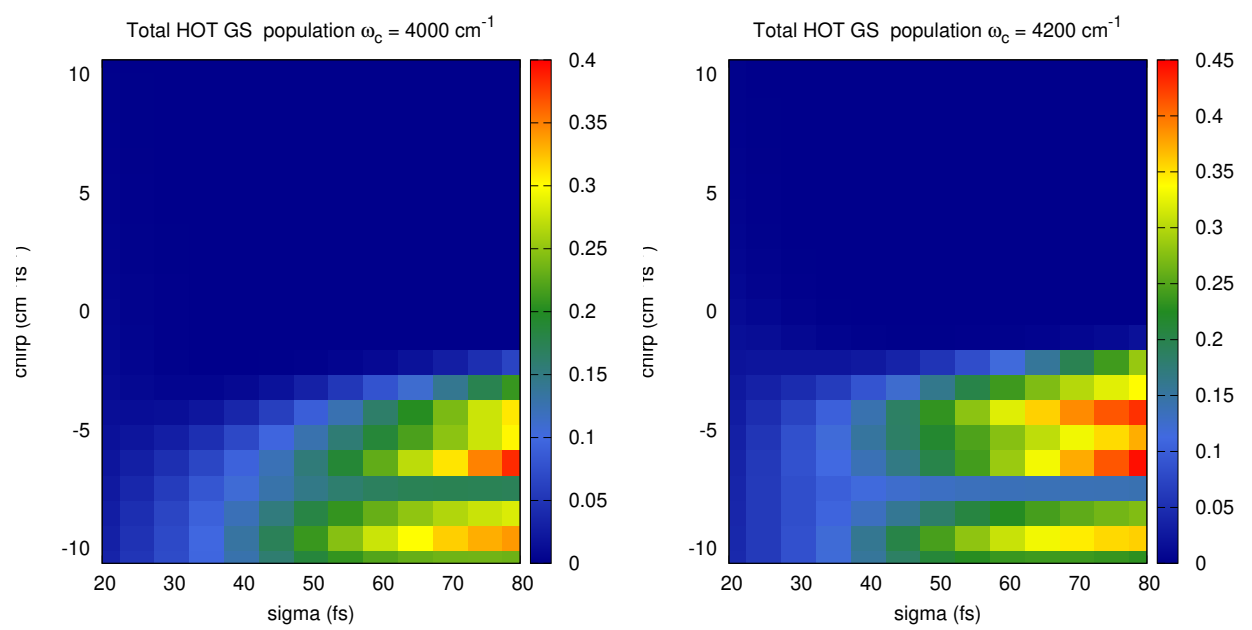

Figure S5: $\omega_{c}=4000 \mathrm{~cm}^{-1}$ (left) and $\omega_{c}=4200 \mathrm{~cm}^{-1}$ (right).
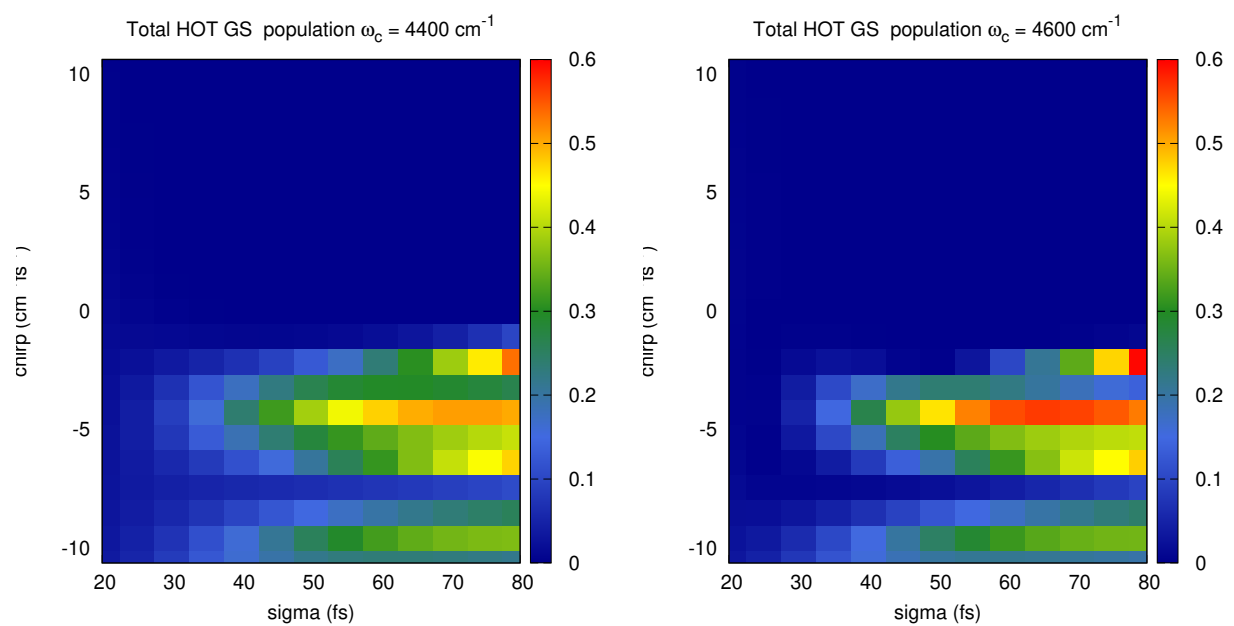

Figure S6: $\omega_{c}=4400 \mathrm{~cm}^{-1}$ (left) and $\omega_{c}=4600 \mathrm{~cm}^{-1}$ (right).
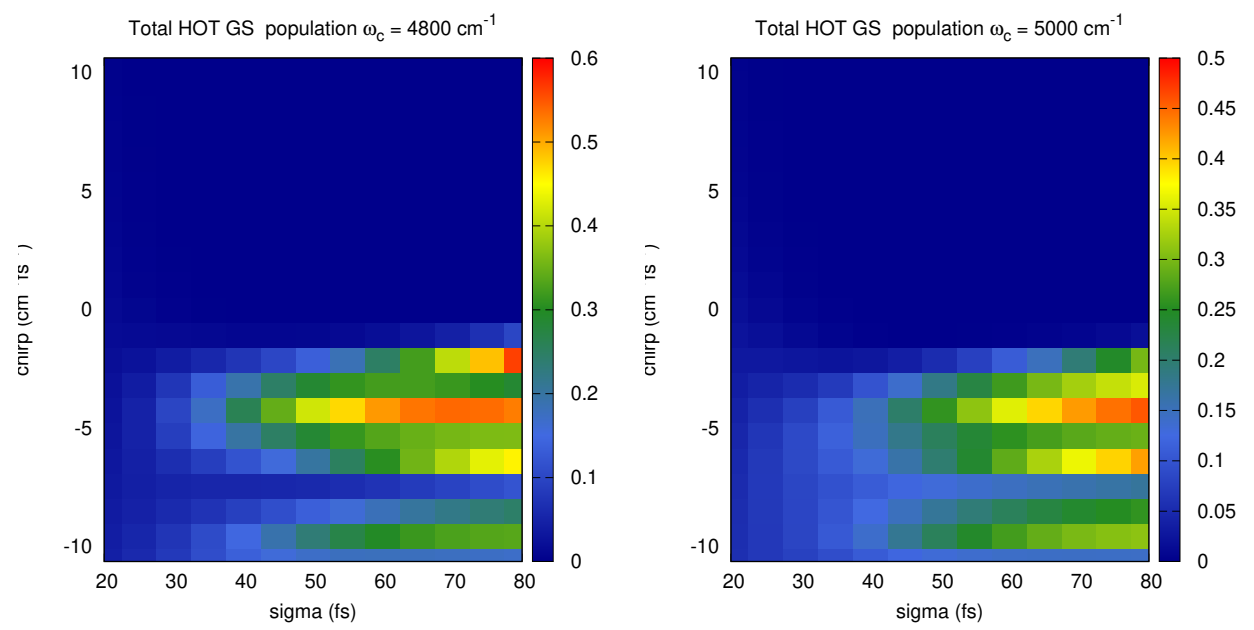

Figure S7: $\omega_{c}=4800 \mathrm{~cm}^{-1}$ (left) and $\omega_{c}=5000 \mathrm{~cm}^{-1}$ (right). 

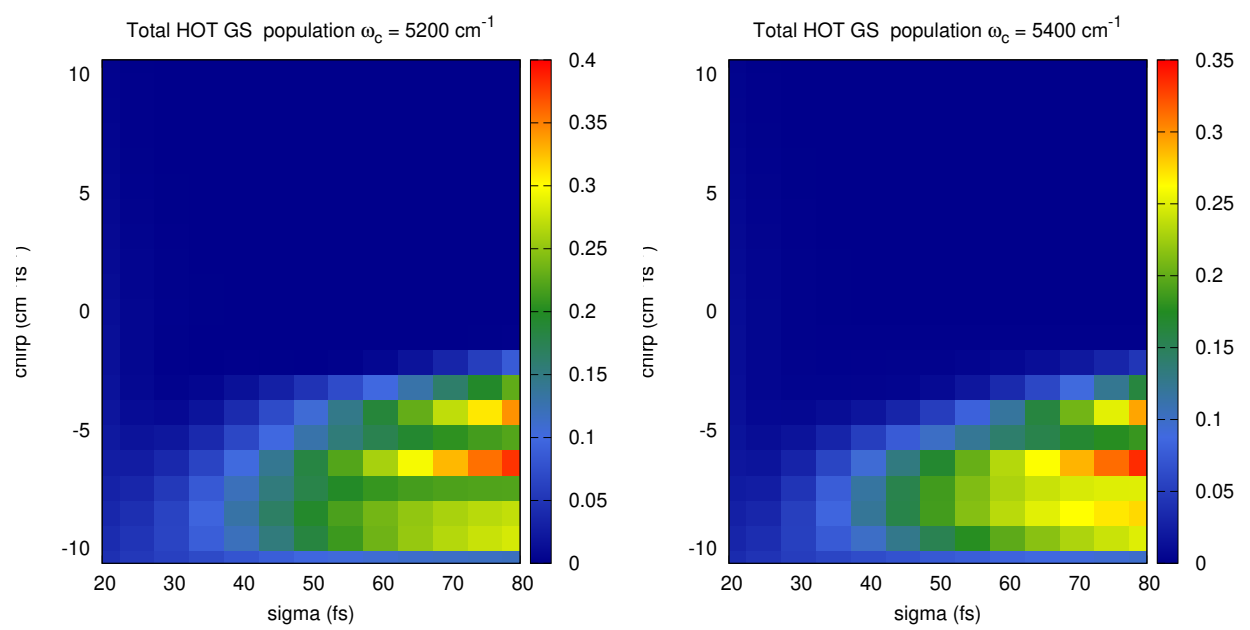

Figure S8: $\omega_{c}=5200 \mathrm{~cm}^{-1}$ (left) and $\omega_{c}=5400 \mathrm{~cm}^{-1}$ (right).
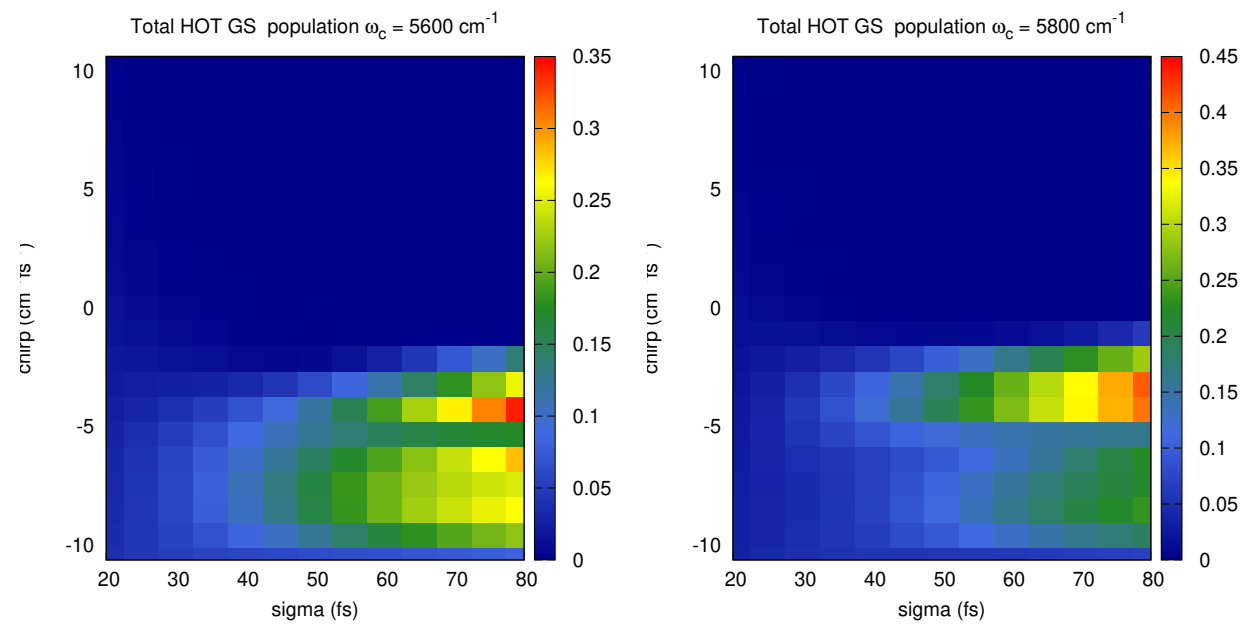

Figure S9: $\omega_{c}=5600 \mathrm{~cm}^{-1}$ (left) and $\omega_{c}=5800 \mathrm{~cm}^{-1}$ (right).

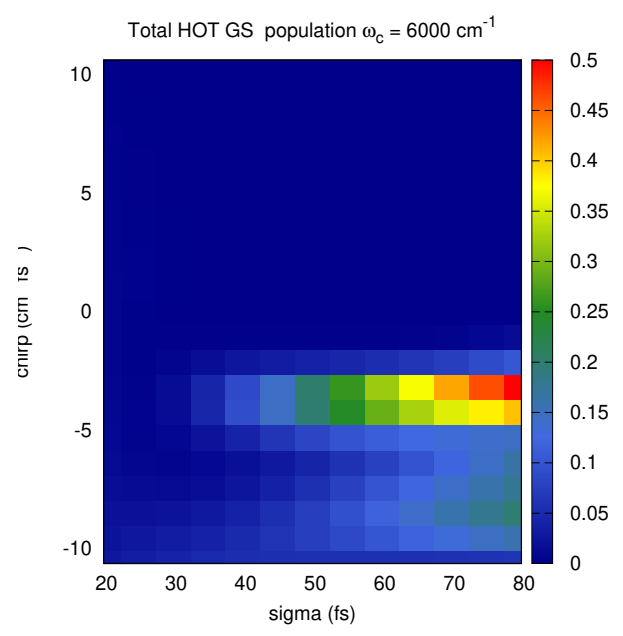

Figure S10: $\omega_{c}=6000 \mathrm{~cm}^{-1}$ 


\section{S6 Comparison of pump-probe spectra at $\mathrm{T}=77 \mathrm{~K}$ and $\mathrm{T}=300 \mathrm{~K}$}

a)

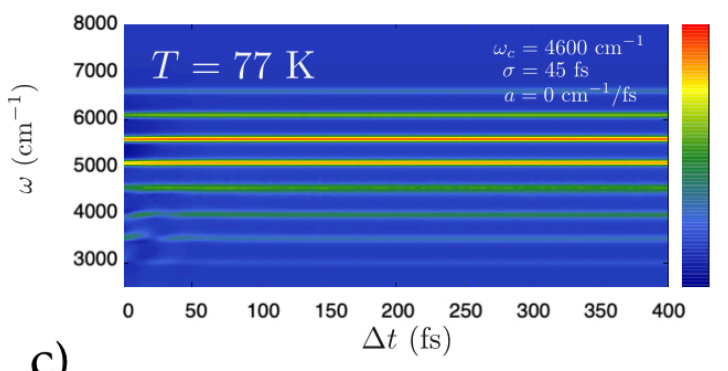

c)

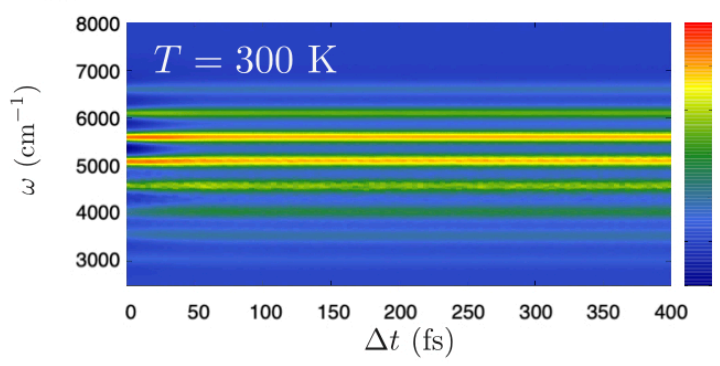

b) Chirped Pulse
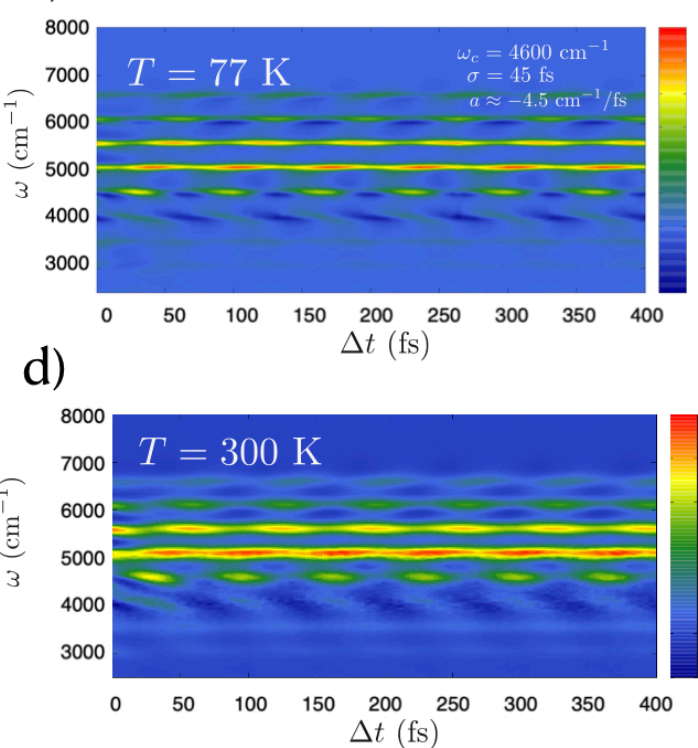

Figure S11: Comparison of pump-probe spectra computed using the simple harmonic model presented in Figure 4 of the main text at $\mathrm{T}=77 \mathrm{~K}$ and $\mathrm{T}=300 \mathrm{~K}$. In all cases here, the bath reorganization energy was chosen as $\lambda=50 \mathrm{~cm}^{-1}$. Panels a) and b) represent the spectra computed at $\mathrm{T}=77 \mathrm{~K}$ with a transform limited and negatively chirped pulse, respectively, while panels c) and d) their respective counterparts computed at $\mathrm{T}=300 \mathrm{~K}$.

To investigate the impact of temperature on the pump-probe signal for the model described in the main text, we computed the signal at $\mathrm{T}=77 \mathrm{~K}$ and $\mathrm{T}=300 \mathrm{~K}$. When $\mathrm{T}$ $=77 \mathrm{~K}$, the quantized $500 \mathrm{~cm}^{-1}$ mode's initial thermal distribution is strongly dominated by the ground vibrational state. When $\mathrm{T}=300 \mathrm{~K}$, there are non-negligible contributions to the thermal distribution from the first excited vibrational state (approximately $8 \%$ ). The spectrum computed at $\mathrm{T}=300 \mathrm{~K}$ was obtained through a weighted sum of the set of non-negligible initial conditions. Since, even at $\mathrm{T}=300 \mathrm{~K}$, the quantized mode's thermal distribution is dominated by the ground vibrational state, there are minimal qualitative differences in the spectra from different temperatures. This increase in temperature is largely manifested as an overall broadening in the spectrum.

\section{References}

[1] Schatz, G. C.; Ratner, M. A. Quantum Mechanics in Chemistry, dover ed.; Dover, 2002.

[2] Abramavicius, D.; Palmieri, B.; Voronine, D. V.; Sanda, F.; Mukamel, S. Chem. Rev. 2009, 109, 2350-2408. 
[3] Provazza, J.; Segatta, F.; Garavelli, M.; Coker, D. F. J. Chem. Theory Comput. 2018, 14, 856-866.

[4] Nitzan, A. Chemical Dynamics in Condensed Phases: Relaxation, Transfer, and Reactions in Condensed Molecular Systems; Oxford University Press, 2006.

[5] Diels, J.-C.; Rudolph, W. Ultrashort Laser Pulse Phenomena; Elsevier, 2006.

[6] Huo, P.; Coker, D. F. J. Chem. Phys. 2012, 137, 22 A535. 